\begin{tabular}{|c|l|}
\hline Title & I solation and structural characterization of magic silver clusters protected by 4-(tert-butyl)benzyl mercaptan \\
\hline Author(s) & Negishi, Y uichi; A rai, Rio; Niihori, Y oshiki; Tsukuda, Tatsuya \\
\hline Citation & $\begin{array}{l}\text { Chemical Communications, 47(20), 5693-5695 } \\
\text { https:/doi.org/40.1039/c0c05587e }\end{array}$ \\
\hline Issue Date & 2011-05-28 \\
\hline Doc URL & http://hdl.handle.net/2115/49263 \\
\hline Rights & Chem. Commun., 2011, 47, 5693-5695- Reproduced by permission of The Royal Society of Chemistry (RSC) \\
\hline Type & article (author version) \\
\hline File Information & Cc47-20_5693-5695.pdf \\
\hline
\end{tabular}

Instructions for use 


\title{
Isolation and structural characterization of magic silver clusters protected by 4-(tert-butyl)benzyl mercaptan
}

\author{
Yuichi Negishi, ${ }^{a}{ }^{a}$ Rio Arai ${ }^{a}$, Yoshiki Niihori ${ }^{a}$ and Tatsuya Tsukuda ${ }^{b}$ \\ Received (in $X X X, X X X) X$ th $X X X X X X X X X 200 X$, Accepted $X$ th $X X X X X X X X X 200 X$ \\ ${ }_{5}$ First published on the web Xth $X X X X X X X X X 200 X$ \\ DOI: $10.1039 / b 000000 x$
}

Small silver clusters (average diameter of $1.2 \mathrm{~nm}$ ) protected by 4(tert-butyl)benzyl mercaptan (BBSH) were converted to stable, monodisperse clusters $(2.1 \mathrm{~nm})$ by a ripening process with excess 10 amount of BBSH. Multiple characterizations of the isolated magic clusters revealed an approximate chemical composition of $\operatorname{Ag}_{\sim 280}(\mathrm{SBB})_{\sim 120}$.

Metal clusters protected by thiolates (M:SR) have attracted considerable attention as building blocks of new functional 15 materials because they exhibit size-specific optical and physical properties. In particular, clusters with high thermodynamic and chemical stability are very promising candidates. Recent intensive studies have revealed a series of magic compositions for Au:SR clusters, such as $\mathrm{Au}_{25}(\mathrm{SR})_{18}, \mathrm{Au}_{38}(\mathrm{SR})_{24}, \mathrm{Au}_{44}(\mathrm{SR})_{28}, \mathrm{Au}_{68}(\mathrm{SR})_{34}$,

$20 \mathrm{Au}_{102}(\mathrm{SR})_{44}$, and $\mathrm{Au}_{144 / 146}(\mathrm{SR})_{59 / 60}{ }^{1-6}$ These magic clusters can be synthesized selectively from the crude mixtures by utilizing their higher tolerance than the others against core etching by thiol molecules. $^{3 b, 3 d, 4 b}$ The origins of the stability as well as the geometric structures of these gold clusters are now well 25 understood. $^{2 d, 4 a, 4 g, 6-8}$

Ag:SR clusters larger than $\sim 5 \mathrm{~nm}$ have been also well studied because of their potential application in surface-enhanced Raman analysis, catalysis and conducting paste. ${ }^{9-17}$ In contrast, little is known about the stable sizes for smaller clusters: reports on the 30 chemical compositions of stable Ag:SR clusters are limited to only two examples, $\mathrm{Ag}_{7}(\mathrm{DMSA})_{4}{ }^{12}$ and $\mathrm{Ag}_{9}(\mathrm{MSA})_{8}{ }^{13 b}$, wherein DMSA and MSA represent meso 2,3-dimercaptosuccinic acid and mercaptosuccinic acid, respectively. Primary reason for this lack of information on stable Ag:SR cluster sizes is that Ag:SR clusters 35 are easily oxidized and are difficult to handle. In order to elucidate their stable cluster sizes, it is important to study on Ag:SR clusters that are not easily oxidized. Murray et al. have reported the formation of $\mathrm{Ag}$ clusters, assigned to $\mathrm{Ag}_{140}(\mathrm{SBB})_{53}$, which show higher resistance to oxidation than other Ag:SR clusters, using 440 (tert-butyl)benzyl mercaptan (BBSH, Scheme S1, ESI $\dagger$ ). ${ }^{10}$ Motivated by this report, in the present work, we searched for magic Ag:SBB clusters by incubating in the presence of BBSH. We were able to convert the as-prepared, polydisperse Ag:SBB clusters into a single Ag:SBB cluster. Multiple characterizations of 45 the magic clusters obtained revealed that their chemical composition is approximately $\mathrm{Ag}_{\sim 280}(\mathrm{SBB})_{\sim 120}$.

Ag:SBB clusters were prepared under Ar atmosphere by the reduction of $\mathrm{Ag}(\mathrm{I})$-SBB polymers, which were formed by the reaction between silver nitrate and BBSH. Transmission electron 50 microscope (TEM) analysis revealed that the as-prepared Ag:SBB clusters (1) have silver cores with average diameters $\left(d_{a v}\right)$ of ca. $1.2 \mathrm{~nm}$ (Fig. S1(a), ESI $\dagger$ ). Cluster 1 was incubated in neat BBSH at $60^{\circ} \mathrm{C}$ under Ar atmosphere. The structures of the products were studied as a function of the incubation time $\left(T_{\text {inc }}\right)$ using TEM, 55 mass spectrometry, optical spectroscopy, gel permeation chromatography (GPC), thermogravimetric analysis (TG), X-ray photoelectron spectroscopy (XPS), and powder X-ray diffraction (XRD). ${ }^{18}$

Figure 1(a) shows positive-ion ${ }^{19}$ electrospray ionization (ESI) 60 mass spectra of Ag:SBB clusters before and after the reaction. A broad peak was observed in the mass spectrum of cluster $\mathbf{1}\left(T_{\mathrm{inc}}=\right.$ $0 \mathrm{~h}$ ) in the mass region of ca. 10 and $15 \mathrm{kDa} .{ }^{20}$ With increasing incubation time, the ion intensity in this region gradually decreased while multiple peaks appeared in the higher mass 65 region. At $T_{\text {inc }}=3 \mathrm{~h}$, clusters with a molecular weight (MW) of $43.5 \mathrm{kDa}$ were formed, and clusters with $\mathrm{MW}=51.8 \mathrm{kDa}$ were additionally produced at $T_{\text {inc }}=6 \mathrm{~h}$. The $51.8 \mathrm{kDa}$ clusters prevailed over the $43.5 \mathrm{kDa}$ clusters with longer incubation time and the latter eventually disappeared at $T_{\mathrm{inc}}=24 \mathrm{~h}$. Further 70 increase in the cluster size was not observed, even at $T_{\text {in }}=96 \mathrm{~h}$.
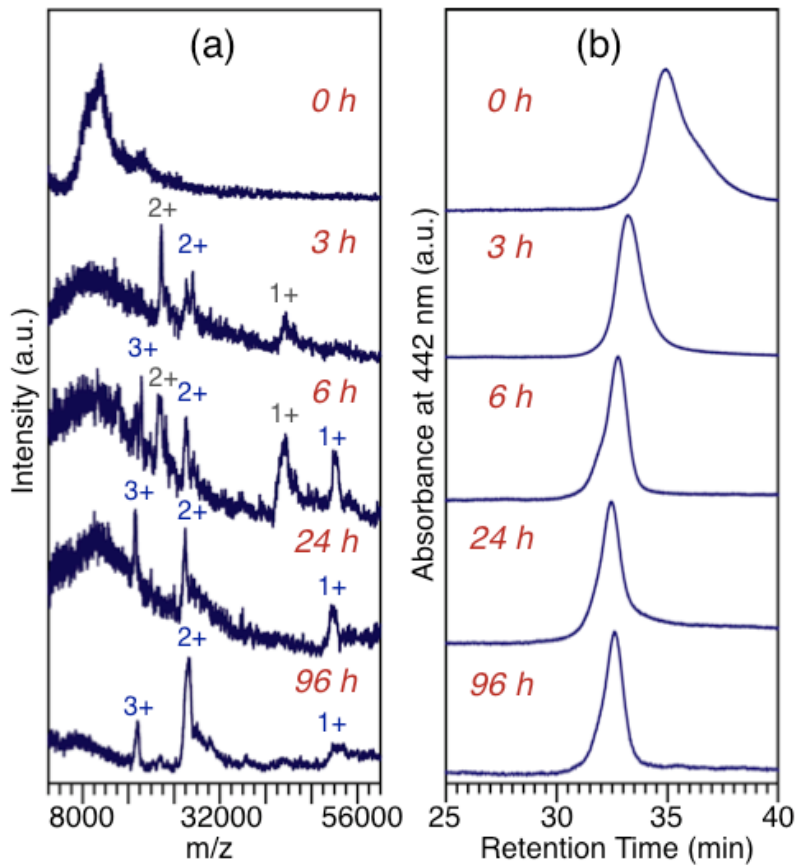

Fig. 1. (a) Positive-ion ESI mass spectra, and (b) GPC chromatograms of Ag:SBB clusters produced by the reaction of $\mathbf{1}$ with BBSH with respect to incubation time. 

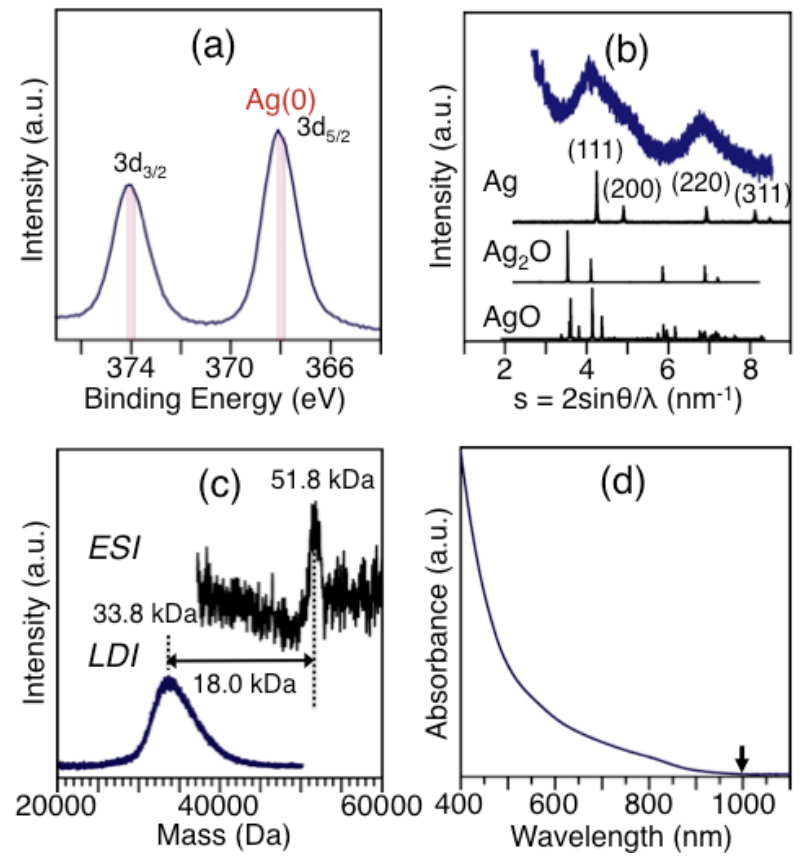

Fig. 2. (a) Ag 3d spectrum, (b) X-ray diffractogram, (c) positive-ion LDI mass spectrum, and (d) optical absorption spectrum of 2. X-ray diffractograms of $\mathrm{Ag}, \mathrm{Ag}_{2} \mathrm{O}$, and $\mathrm{AgO}$ are shown for comparison in panel (b). In panel (c), the top spectrum is the positive ESI mass spectrum of 2.

These results indicate that cluster 1 was converted into $43.5 \mathrm{kDa}$ clusters and finally into $51.8 \mathrm{kDa}$ cluster (2) within $96 \mathrm{~h}$ at $60^{\circ} \mathrm{C}$. The time required for complete conversion of $\mathbf{1}$ into $\mathbf{2}$ depended on the incubation temperature; 16 days and $6 \mathrm{~h}$ at 40 and $80^{\circ} \mathrm{C}$, 5 respectively (Fig. S2, ESI†).

Growth of the Ag clusters during incubation was also confirmed by GPC and TEM. Figure 1(b) shows chromatograms of the products. A broad band was observed at a retention time of $35.5 \mathrm{~min}$ in the chromatogram of cluster $1\left(T_{\mathrm{inc}}=0 \mathrm{~h}\right)$. The 10 retention time of the product gradually reduced with increasing $T_{\text {inc }}$. However, no further shift in the retention time was observed after $96 \mathrm{~h}$, indicating that the increase in particle size was completed within $T_{\text {inc }}=96 \mathrm{~h}$. TEM analysis revealed that cluster 2 has a silver core with a diameter of ca. $2.1 \pm 0.2 \mathrm{~nm}$ (Fig. S1(b), 15 ESI†).

Stability of cluster 2 against oxidation was studied by various methods. Figure 2(a) shows the XPS Ag 3d spectrum of cluster 2. Broad peaks were observed in the spectrum at peak positions attributed to $\mathrm{Ag}(0)$ (367.9 and $373.9 \mathrm{eV}$ ). This indicates that the 20 silver core of cluster $\mathbf{2}$ was not oxidized. The powder XRD pattern of cluster 2 (Fig. 2(b)) indicates the production of the $\mathrm{Ag}(0)$ core. The diffraction patterns of bulk $\mathrm{Ag}, \mathrm{Ag}_{2} \mathrm{O}$, and $\mathrm{AgO}$ are also shown in the figure for comparison. Broad peaks at positions corresponding to diffractions from $\operatorname{Ag}(111),(200)$, (220), and 25 (311) planes are observed for cluster 2 . In contrast, no peaks were observed at positions corresponding to $\mathrm{AgO}$ and $\mathrm{Ag}_{2} \mathrm{O}$, which indicates that the core of cluster $\mathbf{2}$ does not contain oxygen but is composed of only silver. Mass spectrometric analysis showed that cluster $\mathbf{2}$ is stable against oxidation for at least 5 days when stored 30 in air as solid form and dispersed in toluene (Fig. S4 and S5,


Fig. 3. HR-TEM image of 2.

ESI†).

In order to determine the molecular formula of cluster $\mathbf{2}$, we evaluate the number of BBS ligands on the silver core using laser desorption ionization (LDI) mass spectrometry. Figure 2(c) shows 35 the positive ion LDI mass spectrum of cluster 2 . The ESI mass spectrum of cluster $\mathbf{2}$ is also shown for comparison. A broad peak is observed in the vicinity of ca. $33.8 \mathrm{kDa}$ in the LDI mass spectrum. This peak position is shifted by ca. $18 \mathrm{kDa}$ toward the lower mass side as compared to the parent mass peak in the ESI 40 mass spectrum (ca. $51.8 \mathrm{kDa}$ ). For Au:SR clusters, it is known that the C-S bond of the protecting thiolates is selectively and completely dissociated by laser irradiation. ${ }^{21-24}$ Since similar laserinduced dissociation was also reported for Ag:SR clusters, ${ }^{13(b)(c)}$ this decrease in mass can be ascribed to desorption of the 45 arylgroup of SBB due to C-S bond dissociation. A decrease in the mass of $18 \mathrm{kDa}$ corresponds to dissociation of $\sim 120$ BBS aryl groups. Thus, it is considered that the chemical composition of cluster 2 can be approximated as $\mathrm{Ag}_{280}(\mathrm{SBB})_{120}$. The weight ratio of $\mathrm{Ag}$ to BBS obtained by TG analysis $(58.9 \%, 41.1 \%)$ was 50 consistent with that for $\mathrm{Ag}_{\sim 280}(\mathrm{SBB})_{\sim 120}(58.4 \%, 41.6 \%)$. On the basis of these results, we conclude that the stable cluster 2 has a chemical composition of $\mathrm{Ag}_{\sim 280}(\mathrm{SBB})_{\sim 120}$. A similar mass spectrometric analysis of $43.5 \mathrm{kDa}$ clusters revealed that they are expressed as $\mathrm{Ag}_{\sim 223}(\mathrm{SBB})_{\sim 108}$ (Fig. S5, ESI $\dagger$ ).

Optical absorption spectrum of cluster 2 is shown in Figure 2(d). It exhibits absorption onset at ca. $1000 \mathrm{~nm}$ and slight shoulders at ca. 700 and $800 \mathrm{~nm}$, indicating the molecular-like electronic structure of cluster 2. A surface plasmon band (ca. 420 $\mathrm{nm})$ was not observed in contrast to Ag: $\mathrm{SC}_{12}\left(d_{a v}=1.8 \mathrm{~nm}\right){ }^{9}$ This ${ }_{60}$ discrepancy is ascribed either to the difference in thiolates used ${ }^{15}$ or to contribution from larger $\mathrm{Ag}: \mathrm{SC}_{12}$ clusters contained as impurity in ref. 9.

These results reveal that as-prepared Ag:SBB clusters with $d_{a v}$ $=1.2 \mathrm{~nm}$ were focused into stable, monodisperse $\mathrm{Ag}_{\sim 280}(\mathrm{SBB})_{\sim 120}$ 65 via $\mathrm{Ag}_{\sim 223}(\mathrm{SBB})_{\sim 108}$ by a ripening process with excess amount of $\mathrm{BBSH}^{25}{ }^{25}$ It should be stressed here that size focusing via cluster growth is in sharp contrast to that by size reduction of Ag cores in digestive ripening ${ }^{16 \mathrm{~b}, 26}$ and Au cores in core etching. ${ }^{3 b, 3 d, 4 b}$ There are several possible explanations for the selective production of $70 \mathrm{Ag}_{\sim 280}(\mathrm{SBB})_{\sim 120}$. One possibility is that growth of the Ag core is kinetically hindered by the formation of a curved monolayer of BBS; 120 molecules of BBS having a bulky head group may form a monolayer which fit the curvature of an Ag core. Another possibility is intrinsic stability arising from geometrical and 
electronic shell closure. In order to explore this further, we studied the geometrical structure of the Ag core of cluster $\mathbf{2}$ in more detail using high-resolution TEM. Figure 3 shows a representative HRTEM micrograph of cluster 2; Ag particles having 5-fold 5 symmetry can be observed in the HR-TEM micrograph. For $\mathrm{Au}: \mathrm{SR}$ clusters, it has been revealed that the stable clusters consist of the metal core having 5-fold symmetry surrounded by multiple $\mathrm{Au}(\mathrm{I})$-thiolate oligomers. ${ }^{2 d, 4 a, 4 g, 6-8}$ Figure 3 suggests that cluster 2 also has a core with a similar structure to that of stable Au:SR 10 clusters. By assuming the density of bulk $\mathrm{Ag}\left(10.5 \mathrm{~g} / \mathrm{cm}^{3}\right)$, we can estimate the number of the silver atoms in $2.1 \mathrm{~nm}$ cluster to be $\sim 227$. This is smaller than that determined by mass spectrometry and suggests the formation of Ag-thiolate oligomer interface. We must await the single crystal XRD to determine the structure.

15 To summarize, we have succeeded in demonstrating the size convergence of Ag:SBB clusters into a chemically stable cluster. Experiments revealed that the stable cluster has the chemical composition of $\operatorname{Ag}_{\sim 280}(\mathrm{SBB})_{\sim 120}$. This cluster is size-selectively synthesized by reaction with BBSH. It is anticipated that this

20 facilitates their crystallization and full structure determination, which is essential for understanding the origin of the stability and the exceptional physical properties of Ag:SR clusters.

We thank Dr. Hironori Tsunoyama and Mr. Hirokazu Kobayashi for technical assistance and Mr. Wataru Kurashige for 25 their valuable comments. This work was financially supported by Grants-in-Aid for Scientific Research (Grant Nos. 20038045 and 21685003) and the "Nanotechnology Network" from the Ministry of Education, Culture, Sports, Science and Technology (MEXT) of Japan, and the JGC-S Scholarship Foundation.

\section{${ }_{30}$ Notes and references}

${ }^{a}$ Department of Applied Chemistry, Faculty of Science, Tokyo University of Science, 1-3 Kagurazaka, Shinjuku-ku, Tokyo 162-8601, Japan. Fax: +81-3-5261-4631; Tel: +81-3-5228-9145; E-mail: negishi@rs.kagu.tus.ac.jp

${ }_{35}{ }^{b}$ Catalysis Research Center, Hokkaido University, Nishi, 10, Kita 21, Sapporo 001-0021, Japan.

† Electronic Supplementary Information (ESI) available: Experimental procedures and characterization. See DOI: 10.1039/b000000x/

401 (a) T. G. Schaaff and R. L. Whetten, J. Phys. Chem. B, 2000, 104, 2630-2641; (b) R. C. Price and R. L. Whetten, J. Am. Chem. Soc., 2005, 127, 13750-13751.

2 (a) R. L. Donkers, D. Lee and R. W. Murray, Langmuir, 2004, 20, 1945-1952; (b) J. B. Tracy, G. Kalyuzhny, M. C. Crowe, R. 45 Balasubramanian, J.-P. Choi and R. W. Murray, J. Am. Chem. Soc., 2007, 129, 6706-6707; (c) A. Dass, A. Stevenson, G. R. Dubay, J. B. Tracy and R. W. Murray, J. Am. Chem. Soc., 2008, 130, 5940-5946; (d) M. W. Heaven, A. Dass, P. S. White, K. M. Holt and R. W. Murray, J. Am. Chem. Soc., 2008, 130, 3754-3755; (e) C. A. Fields-Zinna, R.

50 Sardar, C. A. Beasley and R. W. Murray, J. Am. Chem. Soc., 2009, 131 16266-16271.

3 (a) Y. Negishi, K. Nobusada and T. Tsukuda, J. Am. Chem. Soc., 2005, 127, 5261-5270; (b) Y. Shichibu, Y. Negishi, H. Tsunoyama, M. Kanehara, T. Teranishi and T. Tsukuda, Small, 2007, 3, 835-839; (c) Y.

55 Negishi, N. K. Chaki, Y. Shichibu, R. L. Whetten and T. Tsukuda, J. Am. Chem. Soc., 2007, 129, 11322-11323; (d) N. K. Chaki, Y. Negishi, H. Tsunoyama, Y. Shichibu and T. Tsukuda, J. Am. Chem. Soc., 2008, 130, 8608-8610.

4 (a) M. Zhu, C. M. Aikens, F. J. Hollander, G. C. Schatz and R. Jin, J. 60 Am. Chem. Soc., 2008, 130, 5883-5885; (b) H. Qian, Y. Zhu and R. Jin, ACS Nano, 2009, 3, 3795-3803; (c) H. Qian and R. Jin, Nano. Lett.,
2009, 9, 4083-4087; (d) M. Zhu, H. Qian and R. Jin, J. Am. Chem. Soc., 2009, 131, 7220-7221; (e) M. Zhu, H. Qian and R. Jin, J. Phys Chem. Lett., 2010, 1, 1003-1007; (f) H. Qian, Y. Zhu and R. Jin, J. Am. Chem. Soc., 2010, 132, 4583-4585; ( ) H. Qian, W. T. Eckenhoff, Y. Zhu, T. Pintauer and R. Jin, J. Am. Chem. Soc., 2010, 132, 8280-8281.

5 A. Dass, J. Am. Chem. Soc., 2009, 131, 11666-11667.

6 P. D. Jadzinsky, G. Calero, C. J. Ackerson, D. A. Bushnell and R. D. Kornberg, Science, 2007, 318, 430-433.

707 (a) M. Walter, J. Akola, O. Lopez-Acevedo, P. D. Jadzinsky, G. Calero, C. J. Ackerson, R. L. Whetten, H. Grönbeck and H. Häkkinen, Proc. Natl. Acad. Sci. U.S.A., 2008, 105, 9157-9162; (b) O. Lopez-Acevedo, J. Akola, R. L. Whetten, H. Grönbeck and H. Häkkinen, J. Phys. Chem. C, 2009, 113, 5035-5038.

758 O. Lopez-Acevedo, H. Tsunoyama, T. Tsukuda, H. Häkkinen and C. M. Aikens, J. Am. Chem. Soc., 2010, 132, 8210-8218.

9 J. P. Wilcoxon, J. E. Martin and P. Provencio, J. Chem. Phys., 2001, 115, 998-1008.

10 M. R. Branham, A. D. Douglas, A. J. Mills, J. B. Tracy, P. S. White and R. W. Murray, Langmuir, 2006, 22, 11376-11383.

11 (a) N. Nishida, H. Yao, T. Ueda, A. Sasaki and K. Kimura, Chem. Mater., 2007, 19, 2831-2841; (b) N. Nishida, H. Yao and K. Kimura, Langmuir, 2008, 24, 2759-2766; (c) H. Yao, M. Saeki, K. Kimura, J. Phys. Chem. C, 2010, 114, 15909-15915.

8512 Z. Wu, E. Lanni, W. Chen, M. E. Bier, D. Ly and R. Jin, J. Am. Chem. Soc., 2009, 131, 16672-16674.

13 (a) K. V. Mrudula, T. U. B. Rao and T. Pradeep, J. Mater. Chem., 2009, 19, 4335-4342; (b) T. U. B. Rao, B. Nataraju and T. Pradeep, J. Am. Chem. Soc., 2010, 132, 16304-16307; (c) T. U. B. Rao and T. Pradeep, Angew. Chem. Int. Ed., 2010, 49, 3925-3929.

14 (a) N. Cathcart, P. Mistry, C. Makra, B. Pietrobon, N. Coombs, M. Jelokhani-Niaraki and V. Kitaev, Langmuir, 2009, 25, 5840-5846; (b) N. Cathcart and V. Kitaev, J. Phys. Chem. C, 2010, 114, 16010-16017.

15 O. M. Bakr, V. Amendola, C. M. Aikens, W. Wenseleers, R. Li, L. D. 95 Negro, G. C. Schatz and F. Stellacci, Angew. Chem. Int. Ed., 2009, 48, 5921-5926.

16 (a) S. Kumar, M. D. Bolan and T. P. Bigioni, J. Am. Chem. Soc., 2010, 132, 13141-13143; (b) R. Shankar, B. B. Wu and T. P. Bigioni, J. Phys. Chem. C, 2010, 114, 15916-15923.

10017 K. Mori, A. Kumami, M. Tomonori and H. Yamashita, J. Phys. Chem. C, 2009, 113 16850-16854.

18 See the Supporting Information.

19 Since BBSH does not contain a binding site for proton, the Ag core of cluster $\mathbf{1}$ is thought to be positively charged. Baselines of the ESI mass spectra are rugged due to the instrumental noise.

20 Although relative population between $\sim 10$ and $\sim 15 \mathrm{kDa}$ clusters changed depending on the batch, the final product obtained by incubation was always $51.8 \mathrm{kDa}$ clusters.

21 (a) M. M. Alvarez, J. T. Khoury, T. G. Schaaff, M. Shafigullin, I. 110 Vezmar and R. L. Whetten, Chem. Phys. Lett., 1997, 266, 91-98; (b) T. G. Schaaff and R. L. Whetten, J. Phys. Chem. B, 1999, 103, 93949396; (c) T. G. Schaaff, M. N. Shafigullin, J. T. Khoury, I. Vezmar and R. L. Whetten, J. Phys. Chem. B, 2001, 105, 8785-8796.

22 R. J. Arnold and J. P. Reilly, J. Am. Chem. Soc., 1998, 120, 1528-1532. 11523 V. L. Jimenez, D. G. Georganopoulou, R. J. White, A. S. Harper, A. J. Mills, D. I. Lee and R. W. Murray, Langmuir, 2004, 20, 6864-6870.

24 Z. Wu, C. Gayathri, R. R. Gil and R. Jin, J. Am. Chem. Soc., 2009, 131, 6535-6542.

25 It is conceivable that the growth could occur after etching of the as120 prepared clusters. However, we observed no sign of etching under the present experimental conditions as seen in Figure 1(a) and (b).

26 (a) B. L. V. Prasad, S. I. Stoeva, C. M. Sorensen and K. J. Klabunde, Langmuir, 2002, 18, 7515-7520; (b) B. L. V. Prasad, S. I. Stoeva, C. M. Sorensen and K. J. Klabunde, Chem. Mater., 2003, 15 (4) 935-942. 